\title{
Machine learning to reveal an astute risk predictive framework for Gynecologic Cancer and its impact on women psychology: Bangladeshi perspective
}

\author{
Sayed Asaduzzaman ${ }^{1,4^{*}} \mathbb{0}$, Md. Raihan Ahmed², Hasin Rehana ${ }^{3,5}$, Setu Chakraborty ${ }^{6}$, Md. Shariful Islam ${ }^{6}$ and \\ Touhid Bhuiyan²
}

${ }^{*}$ Correspondence:

s.asaduzzaman@rmstu.edu.

bd; asadcse.rmstu@gmail.

com; sayed.asaduzzaman.

bd@ieee.org

${ }^{1}$ Department of Computer

Science and Engineering,

Rangamati Science

and Technology University,

Vedvedi, Rangamati,

Bangladesh

Full list of author information

is available at the end of the article

\begin{abstract}
Background: In this research, an astute system has been developed by using machine learning and data mining approach to predict the risk level of cervical and ovarian cancer in association to stress.

Results: For functioning factors and subfactors, several machine learning models like Logistics Regression, Random Forest, AdaBoost, Naïve Bayes, Neural Network, kNN, CN2 rule Inducer, Decision Tree, Quadratic Classifier were compared with standard metrics e.g., F1, AUC, CA. For certainty info gain, gain ratio, gini index were revealed for both cervical and ovarian cancer. Attributes were ranked using different feature selection evaluators. Then the most significant analysis was made with the significant factors. Factors like children, age of first intercourse, age of husband, Pap test, age are the most significant factors of cervical cancer. On the other hand, genital area infection, pregnancy problems, use of drugs, abortion, and the number of children are important factors of ovarian cancer.
\end{abstract}

Conclusion: Resulting factors were merged, categorized, weighted according to their significance level. The categorized factors were indexed using ranker algorithm which provides them a weightage value. An algorithm has been formulated afterward which can be used to predict the risk level of cervical and ovarian cancer in relation to women's mental health. The research will have a great impact on the low incoming country like Bangladesh as most women in low incoming nations were unaware of it. As these two can be described as the most sensitive cancers to women, the development of the application from algorithm will also help to reduce women's mental stress. More data and parameters will be added in future for research in this perspective.

Keywords: Gynecological cancer, Significant risk factors, Smart prediction tool, Women psychology, Machine learning, Data mining author(s) and the source, provide a link to the Creative Commons licence, and indicate if changes were made. The images or other third party material in this article are included in the article's Creative Commons licence, unless indicated otherwise in a credit line to the material. If material is not included in the article's Creative Commons licence and your intended use is not permitted by statutory regulation or exceeds the permitted use, you will need to obtain permission directly from the copyright holder. To view a copy of this licence, visit http:// creativecommons.org/licenses/by/4.0/. The Creative Commons Public Domain Dedication waiver (http://creativecommons.org/publi cdomain/zero/1.0/) applies to the data made available in this article, unless otherwise stated in a credit line to the data. 


\section{Highlights of the research}

- The Research done on the Dataset of most common Gynecological cancer.

- Analysis of data done by Machine Learning approach.

- Outcome of the research indicates the significant factors and significance level.

- An algorithm has been designed based on the results of the analysis and weightage.

- Algorithm based App outcomes the risk prediction level for gynecological cancer.

\section{Introduction}

Neurodegenerative Disease and post awful neurodegenerative issue are considered as a genuine factor for some major illnesses. It is seen that individuals suffering from neurodegenerative disease disorders have a $55 \%$ chance to be affected with cervical cancer $[1,2]$. Women who experienced at least 6 side effects of post-traumatic neurodegenerative disease disorder had a more crucial danger of being affected with ovarian cancer [3]. According to WHO, the second leading disease is cancer, it causes 9.6 million death in 2018 [4]. An uncontrolled increase of irregular cells exceeding their regular territory with the ability to attack or potentially spread to different organs is cancer. Among different types of cancer cervical and ovarian cancer is the most prominent hazard to female's wellbeing [5]. Due to cervical and ovarian cancer every year, over and above 3,00,000 women dies further half a million were diagnosed. Around $5,00,000$ women were affected with cervical cancer every year and 274000 die due to cervical cancer [6].

An assessment [7] expected to perceive risk factors for Lower Limb Lymphedema (LLL) which is a persistent and weakening condition troubling patients who go through lymphadenectomy for gynecologic cancer and to develop a foreseeing model for its happening. To encourage future researches in the field of gynecologic cancer, a model was introduced in a study [8] to predict the risk for psychologic and conduct morbidity. Neurodegenerative disease is linked with diverse neurodegenerative issue, specifically, the pathophysiological importance of pressure in Alzheimer's infection and several diseases. Some previous studies also showed that neurodegenerative disease spurs on cervical and ovarian cancer $[9,10]$. National Cancer Institute summarizes that cervical tumor constitutes in the cervix, a body part associating with uterus and vagina [11, 12] Human Papillomavirus (HPV) is the main reason behind cervical cancer [13] For the well-known impact of HPV on cervical cancer, an examination [14] was made which audits the articles based on the information on HPV and cervical cancer among Malaysian inhabitants before and after the usage of HPV antibody programs. In [15] a research has been conveyed a study where he found cervical slowly develops without showing any indication at the beginning seemingly hard to discover but can be noticed with respective pap tests. A study was made with 70 patients in china with insomnia provoked by cervical cancer [16]. This irregularly checked preliminarily selected patients with sleep deprivation that arises or exacerbated by cervical disease [17]. A survey was carried out in [16] using the data of Nurses' Health Study found a substantial relationship between treatment for PTSD (Post Traumatic Stress Disorder) and the growth of ovarian cancer. According to American Cancer Institute, ovarian cancer is supposed to start in the 
ovaries but recent knowledge exhibits that numerous ovarian tumors may begin in the fallopian tubes, which holds two ovaries after the body of the uterus. Likely cervical, ovarian cancer is hard to recognize [18]. The ovaries lie profound inside the abdominopelvic pit, making them hard to view or feel $[19,20]$. The epithelial ovarian disease stays an exceptionally dangerous (Hunn \& Rodriguez, 2012). A feasible study [20] offers risk management options of screening and prevention of ovarian cancer. It has been proved, due to the amendment of the p53 gene, cells affected due to neurodegenerative diseases persuades ovarian epithelial cancer [21]. A study [22] presents data which shows, emission of incendiary proteins in ovarian cancer cell were prompted by stress hormones.

According to [23], Cancer is the main source of death. Recent studies [15] suggested that the event of lung cancer has expanded quickly and turned the most widely recognized disease worldwide. A full research was made in [24] to build up a framework that can be utilized by an individual to test his risk level of Lung Cancer. And utilizing the acquired knowledge an experiment was able to predict the risk level of lung cancer [25]. Another research was carried out to build up a system that can be utilized by an individual for knowing his risk level of skin cancer [26]. Presently Type-1 Diabetes is also a shocking sickness in Bangladesh. Type 1 diabetes, which is known as adolescent diabetes or insulin-subordinate diabetes, is an interminable condition where the pancreas delivers mostly zero insulin. Information has been gathered from Dhaka dependent on a particular questionnaire to show the association and criticalness among the degree of elements [27].

In this paper "Introduction" section, represents the risk prediction models and their techniques behind prediction were discussed elaborately. We conduct experiments on three datasets in "Material and methods" section and it was conducted with the help of knowledge discovery. Their efficiency in prediction is shown with a set of figures and tables. This section also contains the output of our research which is the mobile application that we have prepared for risk prediction. For preparing this application at first, we've made an equation to differentiate the risk level and prepared an algorithm. The algorithm is provided in "Material and methods" section. Finally, this work is concluded in "Results and discussion" section and future work is proposed afterward.

\section{Material and methods}

This paper uses popular data mining and machine learning models were compared with metrics such as accuracy, precision, recall, F1, support [28] This was found using a sklearn library [29] of python and orange machine learning and data mining toolkit. We've further proposed an equation based on the difference between the result-metrics of these two toolkits. Using apriori algorithm correlation among the significant factors which describe the dependency among the factors were described. Ranker algorithm is the most efficient algorithm can be used to rank the features for indexing than BestFirst or, GreedyStepwise. Feature selection was performed using the ranker algorithm. Key factors on the data analysis were derived for all the evaluators of the ranker algorithm [30]. Afterward, it was compared among them and the worthiest attribute was obtained. For prediction, it is important to find out the significant factors. Here, the importance of factors has been gathered according to info gain, gain ratio, gini index [31, 32]. 


\section{Data collection}

In total 866 data were collected from various diagnosis centers of patients suffering from diseases like ovarian, cervical, and stress (Mental) disorder. Data of 161 female patients were collected from those who were experiencing cervical cancer. Data were collected from a set of questionnaires which includes 25 attributes. 522 patients of ovarian cancer were interviewed with set questions which contain 46 risk factors. The set of Questioner for Cervical Cancer and Ovarian Cancer has been provided as (Additional File 1 and Additional File 2) Respectively.

\section{Data preprocessing}

Data cleaning, data integration, data selection, data transformation are four leading tasks of data pre-processing to convert the dataset from noisy, inconsistent data to a format suitable for mining and learning predictability. The corrupted or distorted data with meaningless information those are provided by the patients while answering the questionnaire are noisy data. In the data cleaning phase those noisy, conflicting and inconsistent data were removed. Valuable data were joined in the data integration phase. Data suited for the analysis were retrieved from the dataset in the data selection phase. Finally, in data transformation, data were converted to proper structures which fits for data mining and machine learning analysis. To ignore the collision of the data a small amount of data was altered.

\section{Evaluation of the performance of machine learning models}

In this lesson, eight classifiers are known as SVM, Random Forest, Logistic Regression, AdaBoost, Naïve Bayes, Neural Network, kNN, CN2 rule Inducer were used for evaluation with orange and almost 10 classifiers namely SVM, Random Forest, Logistic Regression, AdaBoost, Naïve Bayes, Neural Network, kNN, Gaussian Process, Decision Tree, Quadratic Classifier were used for assessment of machine learning models. In this context, the performance was measured using standard metrics like the area under the ROC curve (AUC), precision, classification accuracy, recall, specificity, F measure, support. A decision tree was constructed with the important factors of cervical, ovarian, and stress datasets.

Performance measures: Classification accuracy rates for the datasets were analyzed. For each dataset, two classes were identified namely positive and negative. There are four possibilities for a single prediction e.g., true positive, true negative, false positive, false negative. True positive and true negatives are described as how many correct predictions were made. False-positive and false-negative provides how many incorrect predictions were made of positive and negative classes when they belong to positive and negative classes.

Accuracy: It defines the number of correct predictions that were correctly classified from the total number of predictions in ratio Eq. (1)

$$
\text { accuracy }=\frac{T P+T N}{T P+T N+F P+F N}
$$


Precision: Explains the number of positive predictions that were correctly classified by the classifier from the total number of positive predictions Eq. (2)

$$
\text { precision }=\frac{T P}{T P+F P}
$$

Recall: Characterizes the fraction of correct positive predictions of the whole equation Eq. (3)

$$
\text { recall }=\frac{T P}{T P+F N}
$$

F-measure: It predicts the average value of precision and recalls Eq. (4)

$$
F-\text { measure }=\frac{2 \times(\text { precision } \times \text { recall })}{\text { precision }+ \text { recall }}
$$

Specificity: Measures the number of whole negative prediction those are correctly identified by the classifier Eq. (5)

$$
\text { Specificity }=\frac{T N}{T N+F P}
$$

Support: Defines the number of datasets that were analyzed after training and splitting the whole dataset. From my analysis, it is seen that sklearn uses only $24 \%$ of the whole dataset that was used Eq. (6)

$$
\text { Support }=0.24 \times \text { no. of data } \in \text { the dataset }
$$

The first and foremost concept to judge the probability is to find significant factors through various analyses. The study was undertaken with lots of derivatives and algorithms to find out the significant factors. The level of important factors was acquired using information gain, gain ratio, gini index. Information Gain: It is a measurement of the decrease in uncertainty. It is estimated from entropy. Entropy is the measurement of the probability of changeability of the processed information. The higher the entropy, the harder it is to make any determinations from that data Eq. (7)

$$
\begin{aligned}
\operatorname{entropy}\left(p_{1}, p_{2} \ldots p_{n}\right) & =-p_{1} \log p_{1}-p_{2} \log p_{2} \ldots \ldots-p_{n} \log p_{n} \\
E(x) & =\sum_{i=1}^{c}-p_{i} \log p_{i} \\
E(T, X) & =\sum_{c \in X} P(c) E(c)
\end{aligned}
$$

Summation of the feature probability of values times the log probability of the same label. By deducting the value of label and features from the entropy of label information gain is obtained Eq. (8)

$$
\operatorname{Gain}(T, X)=E(T)-E(T, X)
$$


Gain Ratio: Modifies information gain by taking ignored information into account including the number and sizes of the branches that reduces the bias of information gain Eqs. $(9,10)$

$$
\begin{aligned}
& \operatorname{SplitEntropy}(T, X)=\sum_{c \in X}-\frac{T_{x}}{T} \log \frac{T_{x}}{T} \\
& \text { GainRatio }=\frac{\operatorname{Gain}(S, A)}{\operatorname{SplitEntropy}(S, A)}
\end{aligned}
$$

Gini Index: It measures the impurity of a single feature. It is obtained by subtracting the sum of squared probabilities from one Eqs. $(11,12)$

$$
\begin{aligned}
& \operatorname{Gini}(X)=1-\sum_{i=1}^{c}\left(p_{i}\right)^{2} \\
& \text { Pr ediction difference }=\left(\sum \text { highest }-\sum \text { lowest }\right) \div 4
\end{aligned}
$$

From information gain, we acquired the certainty of individual features for a specific label. Gain ratio provides us the same including the intrinsic information of the dataset. Gini index provides how much filthy an individual factor is. All of these values are gathered in terms of 0 and 1. Equation analyzing the chi-square test and results of feature

Table 1 Attribute values for info gain, gain ratio, Gini index, Chi square and ranker value

\begin{tabular}{llll}
\hline Attributes & Info. gain & Gain ratio & Gini index \\
\hline Problem during pregnancy & 0.408 & 0.414 & 0.25 \\
Abortion & 0.355 & 0.355 & 0.225 \\
Have infection in the genital area & 0.342 & 0.356 & 0.213 \\
Affected by breast cancer & 0.251 & 0.278 & 0.157 \\
Estrogen pill taken after menopause & 0.237 & 0.291 & 0.142 \\
Ever had a hysterectomy & 0.228 & 0.268 & 0.141 \\
Condom/diaphrame & 0.296 & 0.385 & 0.182 \\
Any birth control pill & 0.221 & 0.232 & 0.143 \\
Use napkin & 0.22 & 0.263 & 0.135 \\
Pregnency after 35 & 0.212 & 0.245 & 0.133 \\
Pap test & 0.211 & 0.246 & 0.132 \\
Children after 35 & 0.208 & 0.237 & 0.131 \\
Takes hormone after menopause & 0.206 & 0.251 & 0.127 \\
Take adequate fruit & 0.185 & 0.187 & 0.122 \\
Age & 0.545 & 0.312 & 0.302 \\
Oral contraception & 0.253 & 0.316 & 0.158 \\
Education & 0.192 & 0.102 & 0.113 \\
Age of husband & 0.579 & 0.372 & 0.314 \\
Cancer vaccine taken & 0.007 & 0.031 & 0.005 \\
First sex age & 0.453 & 0.453 & 0.219 \\
Marital status & 0.041 & 0.099 & 0.017 \\
No of children & 0.634 & 0.481 & 0.321 \\
No of sex partner & 0.065 & 0.115 & $0.029+$ A1:D24 \\
\hline
\end{tabular}


selection evaluators we found out the most significant factors which are working behind cervical and ovarian cancer in connection with stress. Then these factors were given different scores based on their significance level. Afterward, the Eq. 12. was defined to separate the risk levels of an individual.

\section{Results and discussion}

The results and discussion section has been discussed and analyzed in this section. Some data mining and machine learning techniques have been applied. We have analyzed the datasets of cervical and ovarian and found common pattern significant attributes. The attributes were selected as common and highly significant factors and correlated with the possibilities of Cervical or Ovarian cancer. Table 1 shows the values of Info Gain, Gini Index, and the Gain ratio of the parameters. Table 1 also shows the Chi-Square test values along with ranking values. Chi-square values were acquired from statistical analysis and ranked values are found from the Data Mining algorithm. A different analysis of the parameters was conducted with different attribute evaluator and has been shown in Table 2.

The serial position of the attributes in different attributes refers to the position of the attribute in the ranking table of corresponding sub evaluators.

Figure 1 indicated the logistic regression analysis values of the actual and predicted data. In $\mathrm{x}$-axis total of 25 values represents 25 separate parameters and the serial of the

Table 2 Attribute values for different algorithm ranking with various sub-evaluator

\begin{tabular}{|c|c|c|c|c|c|}
\hline \multirow[t]{2}{*}{ SerialRank } & \multirow[t]{2}{*}{ Parameters } & \multirow[t]{2}{*}{ Rel Att Eval } & \multirow{2}{*}{$\begin{array}{l}\text { Sym Un Att } \\
\text { Eval }\end{array}$} & \multirow{2}{*}{$\begin{array}{l}\text { Info Gain Att } \\
\text { Eval }\end{array}$} & \multirow{2}{*}{$\begin{array}{l}\text { OneR } \\
\text { Att } \\
\text { Eval }\end{array}$} \\
\hline & & & & & \\
\hline 1 & Problem during pregnancy & 1 & 1 & 1 & 1 \\
\hline 2 & Abortion & NA & 3 & 3 & 3 \\
\hline 3 & Have infection in the genital area & 2 & 2 & 2 & 2 \\
\hline 4 & Affected by breast cancer & 4 & 4 & 4 & 4 \\
\hline 5 & Estrogen pill taken after menopause & 15 & 5 & 5 & 9 \\
\hline 6 & Ever had a hysterectomy & 12 & 6 & 6 & 15 \\
\hline 7 & Condom or diaphragm & 7 & 7 & 7 & 6 \\
\hline 8 & Any birth control pill & 5 & 10 & 9 & 5 \\
\hline 9 & Use napkin & 9 & 11 & 11 & 12 \\
\hline 10 & Pregnancy after 35 & 14 & 12 & 14 & 10 \\
\hline 11 & Pap test & 18 & 15 & 15 & 7 \\
\hline 12 & Children after 35 & 10 & 16 & 17 & 14 \\
\hline 13 & Takes hormone after menopause & 19 & 17 & 18 & 18 \\
\hline 14 & Take adequate fruit & 17 & 19 & 19 & 17 \\
\hline 15 & Age & 12 & 18 & 20 & 19 \\
\hline 16 & Oral contraception & 11 & 20 & 21 & 21 \\
\hline 17 & Education & NA & 21 & 22 & 20 \\
\hline 18 & Age of husband & NA & 22 & 24 & 22 \\
\hline 19 & Cancer vaccine taken & NA & 24 & NA & 24 \\
\hline 20 & First sex age & 21 & NA & NA & NA \\
\hline 21 & Marital status & NA & NA & NA & NA \\
\hline 22 & Number of children & 20 & NA & NA & NA \\
\hline 23 & Number of sex partner & 22 & NA & NA & NA \\
\hline 24 & Long term pressure & 24 & NA & NA & NA \\
\hline
\end{tabular}




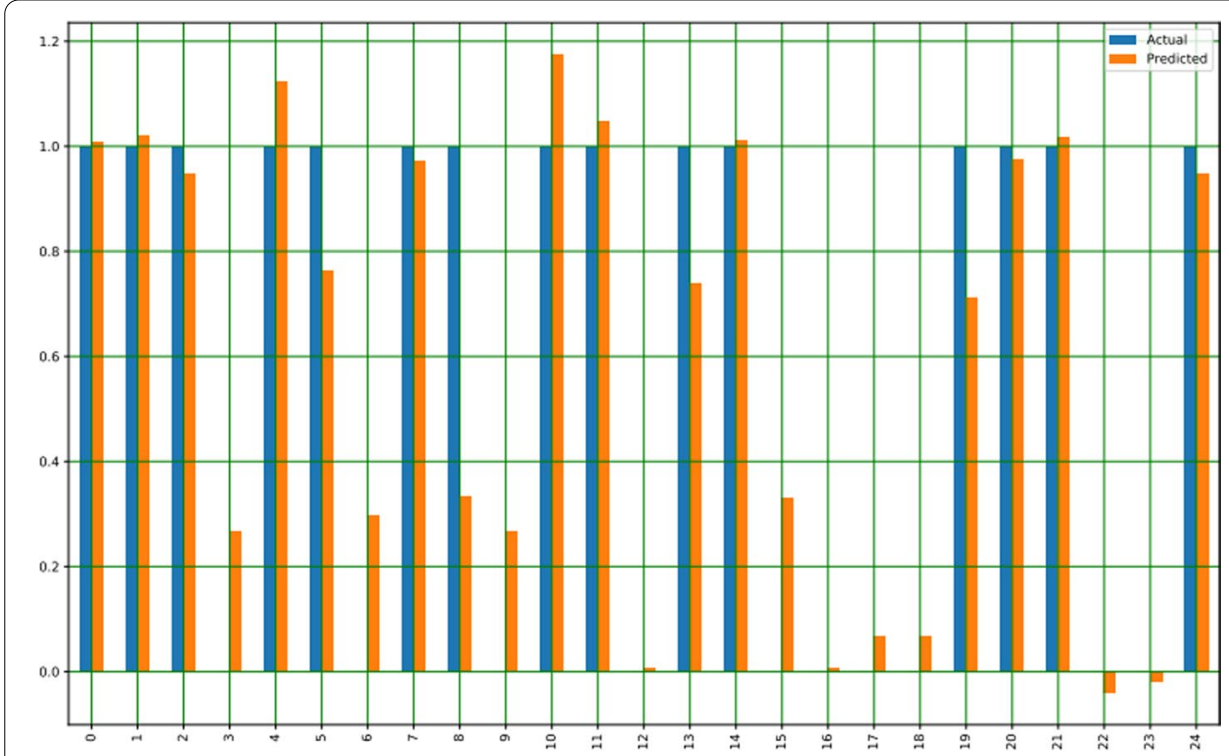

Fig. 1 Linear regression probabilities for different parameters

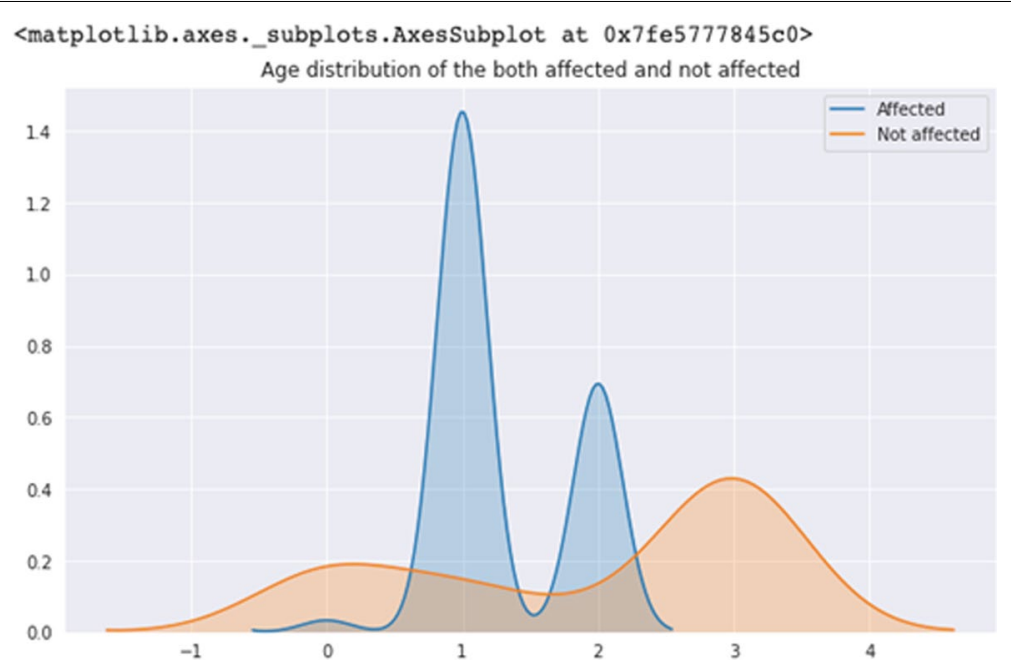

Fig. 2 KDE Plot for both affected and not affected people according to age distribution

parameters are as same as the serial of Table 2. The 24th parameter is related to the mental health or stress of women. The following figure depicts that the higher no of affected women has been shown by linear logistic regression. KDE plot of Fig. 2 tends to estimate the probability distribution functions of affected and non-affected women. The sub-parameters were assigned numeric values e.g., 1 represents above 60, 2 represents 46-60 etc. The affected plot varies from 1 to 1.45 means a higher number of affected women rely on age more than 60 and similarly, 2-0.7 points describe that the second most affected women had an age of 46-60. Violin plot visualizes the distribution of data and its probability density as shown in Fig. 3. Children of 3-5 or above 5 suffer from 


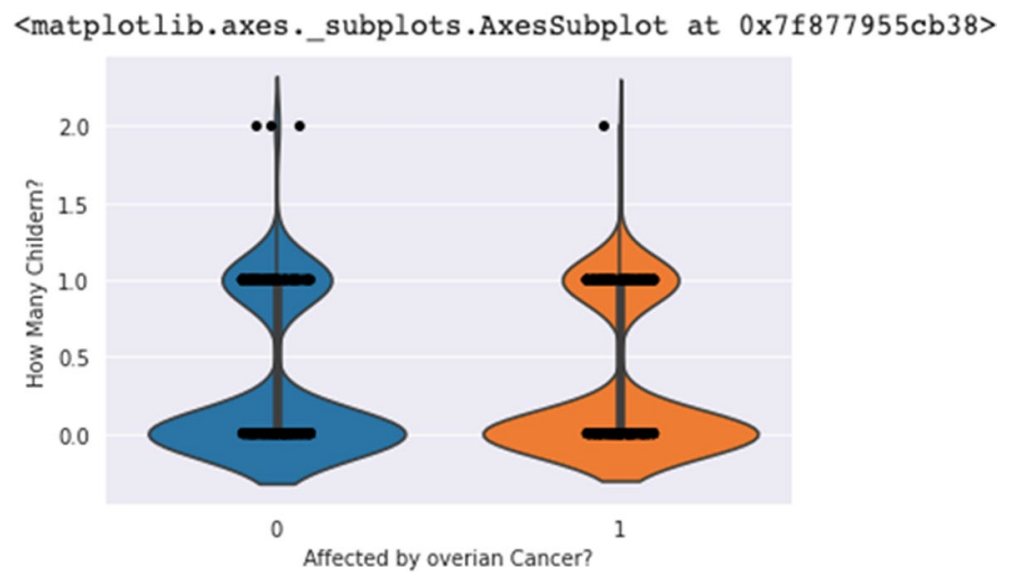

Fig. 3 Violin plot for ovarian cancer versus no of children

Table 3 Accuracy of stress according to different machine learning algorithms

\begin{tabular}{lllllllll}
\hline Model & Tool & AUC & CA & F1 & Precision & Recall & Specificity & Support \\
\hline SVM & Orange & 0.746 & 0.744 & 0.698 & 0.736 & 0.744 & 0.462 & - \\
& Sklearn & 0.615 & & 0.6 & 0.59 & 0.62 & - & 39 \\
Random & Orange & 0.746 & 0.725 & 0.707 & 0.705 & 0.725 & 0.525 & - \\
Forest & Sklearn & 0.692 & & 0.7 & 0.73 & 0.69 & - & 39 \\
Logistic & Orange & 0.796 & 0.769 & 0.752 & 0.757 & 0.769 & 0.579 & - \\
Regression & Sklearn & 0.769 & & 0.77 & 0.76 & 0.77 & - & 39 \\
AdaBoost & Orange & 0.744 & 0.788 & 0.785 & 0.783 & 0.788 & 0.683 & - \\
& Sklearn & 0.79 & & 0.79 & 0.79 & 0.79 & - & 39 \\
Naïve & Orange & 0.753 & 0.763 & 0.763 & 0.763 & 0.763 & 0.672 & - \\
Bayes & Sklearn & 0.785 & & 0.78 & 0.79 & 0.78 & - & 39 \\
Neural & Orange & 0.767 & 0.75 & 0.747 & 0.745 & 0.75 & 0.631 & - \\
Network & Sklearn & 0.692 & & 0.57 & 0.48 & 0.69 & - & 39 \\
kNN & Orange & 0.646 & 0.669 & 0.627 & 0.618 & 0.669 & 0.394 & - \\
& Sklearn & 0.615 & & 0.6 & 0.59 & 0.62 & - & 39 \\
CN2 rule & Orange & 0.721 & 0.694 & 0.696 & 0.699 & 0.694 & 0.595 & - \\
Inducer & Sklearn & & - & & - & & - & - \\
Decision & Orange & 0.7 & 0.713 & 0.717 & 0.723 & 0.713 & 0.639 & - \\
Tree & Sklearn & 0.692 & & 0.7 & 0.71 & 0.69 & - & 39 \\
Quadratic & Orange & & - & & - & & - & - \\
Classifier & Sklearn & 0.744 & & 0.7 & 0.75 & 0.74 & - & 39 \\
\hline
\end{tabular}

cervical or ovarian cancer according to the violin plot of Fig. 3. Violin of 0 level means having a higher number of children which had those cancers.

Tables 3, 4 and 5 shows the accuracy of the data for Mental Stress, Ovarian cancer and Cervical cancer according to different machine learning classifiers and also displays the classification accuracy, F1, precision metrics which can be used to compare the machine leaning models. The accuracy level is organized between 0 and 1 . The prediction accuracy of the model increases with the value getting closer to 1 . It also indicates the significance. All the significant factors and sub-factors of the diseases were first indexed with the help of ranker algorithm and then combined to get a whole picture which is later 
Table 4 Accuracy of ovarian cancer according to different machine learning algorithms

\begin{tabular}{lllllllc}
\hline Model & Tool & AUC & CA & F1 & Precision & Recall & Specificity \\
\hline SVM & Orange & 0.883 & 0.742 & 0.704 & 0.741 & 0.742 & 0.8 \\
& Sklearn & 0.861 & - & 0.85 & 0.87 & 0.85 & - \\
Random & Orange & 0.868 & 0.755 & 0.745 & 0.744 & 0.755 & 0.841 \\
Forest & Sklearn & 0.972 & - & 0.97 & 0.97 & 0.98 & - \\
Logistic & Orange & 0.863 & 0.735 & 0.721 & 0.72 & 0.735 & 0.828 \\
Regression & Sklearn & 1 & - & 1 & 1 & 1 & - \\
AdaBoost & Orange & 0.86 & 0.742 & 0.737 & 0.737 & 0.742 & 0.828 \\
& Sklearn & 1 & - & 1 & 1 & 1 & - \\
Naïve & Orange & 0.851 & 0.621 & 0.627 & 0.642 & 0.621 & 0.836 \\
Bayes & Sklearn & 0.958 & - & 0.96 & 0.96 & 0.96 & - \\
Neural & Orange & 0.847 & 0.718 & 0.719 & 0.721 & 0.718 & 0.838 \\
Network & Sklearn & 0.986 & - & 0.99 & 0.99 & 0.98 & - \\
kNN & Orange & 0.845 & 0.735 & 0.725 & 0.723 & 0.735 & 0.833 \\
& Sklearn & 0.861 & - & 0.85 & 0.87 & 0.85 & - \\
CN2 rule & Orange & 0.821 & 0.674 & 0.675 & 0.676 & 0.674 & 0.815 \\
Inducer & Sklearn & - & - & - & - & - & - \\
Decision & Orange & - & - & - & - & - & - \\
Tree & Sklearn & 0.986 & - & 0.99 & 0.98 & 0.99 & - \\
Quadratic & Orange & - & - & - & - & - & - \\
Classifier & Sklearn & 0.431 & - & 0.3 & 0.22 & 0.5 & - \\
\hline
\end{tabular}

Table 5 Accuracy of cervical cancer according to different machine learning algorithms

\begin{tabular}{|c|c|c|c|c|c|c|c|c|}
\hline Model & Tool & AUC & CA & F1 & Precision & Recall & Specificity & Support \\
\hline \multirow[t]{2}{*}{ SVM } & Orange & 0.883 & 0.742 & 0.704 & 0.741 & 0.742 & 0.8 & - \\
\hline & Sklearn & 0.861 & - & 0.85 & 0.87 & 0.85 & - & 72 \\
\hline \multirow[t]{2}{*}{ Random forest } & Orange & 0.868 & 0.755 & 0.745 & 0.744 & 0.755 & 0.841 & - \\
\hline & Sklearn & 0.972 & - & 0.97 & 0.97 & 0.98 & - & 72 \\
\hline \multirow[t]{2}{*}{ Logistic regression } & Orange & 0.863 & 0.735 & 0.721 & 0.72 & 0.735 & 0.828 & - \\
\hline & Sklearn & 1 & - & 1 & 1 & 1 & - & 72 \\
\hline \multirow[t]{2}{*}{ AdaBoost } & Orange & 0.86 & 0.742 & 0.737 & 0.737 & 0.742 & 0.828 & - \\
\hline & Sklearn & 1 & - & 1 & 1 & 1 & - & 72 \\
\hline \multirow[t]{2}{*}{ Naïve Bayes } & Orange & 0.851 & 0.621 & 0.627 & 0.642 & 0.621 & 0.836 & - \\
\hline & Sklearn & 0.958 & - & 0.96 & 0.96 & 0.96 & - & 72 \\
\hline \multirow[t]{2}{*}{ Neural network } & Orange & 0.847 & 0.718 & 0.719 & 0.721 & 0.718 & 0.838 & - \\
\hline & Sklearn & 0.986 & - & 0.99 & 0.99 & 0.98 & - & 72 \\
\hline \multirow[t]{2}{*}{ kNN } & Orange & 0.845 & 0.735 & 0.725 & 0.723 & 0.735 & 0.833 & - \\
\hline & Sklearn & 0.861 & - & 0.85 & 0.87 & 0.85 & - & 72 \\
\hline \multirow[t]{2}{*}{ CN2 rule inducer } & Orange & 0.821 & 0.674 & 0.675 & 0.676 & 0.674 & 0.815 & - \\
\hline & Sklearn & - & - & - & - & - & - & 72 \\
\hline \multirow[t]{2}{*}{ Decision tree } & Orange & - & - & - & - & - & - & - \\
\hline & Sklearn & 0.986 & - & 0.99 & 0.98 & 0.99 & - & 72 \\
\hline \multirow[t]{2}{*}{ Quadratic classifier } & Orange & - & - & - & - & - & - & - \\
\hline & Sklearn & 0.431 & - & 0.3 & 0.22 & 0.5 & - & 72 \\
\hline
\end{tabular}


Table 6 Weightage value of parameters

\begin{tabular}{|c|c|c|}
\hline Parameters & Sub-parameters & $\begin{array}{l}\text { Weight/ } \\
\text { score }\end{array}$ \\
\hline \multirow[t]{2}{*}{ Problem during pregnancy } & Yes & 14 \\
\hline & No & 13 \\
\hline \multirow[t]{3}{*}{ Abortion } & Yes & 13 \\
\hline & No & 12 \\
\hline & No & 11.5 \\
\hline \multirow[t]{2}{*}{ Affected by breast cancer } & Yes & 11.5 \\
\hline & No & 10.5 \\
\hline \multirow[t]{2}{*}{ Estrogen pill taken after menopause } & No & 10 \\
\hline & Yes & 10.5 \\
\hline \multirow[t]{2}{*}{ Ever had a hysterectomy } & No & 10 \\
\hline & Yes & 9.5 \\
\hline \multirow[t]{2}{*}{ Condom or diaphragm } & No & 9.5 \\
\hline & Yes & 9 \\
\hline \multirow[t]{2}{*}{ Any birth control pill } & No & 8.5 \\
\hline & Yes & 9 \\
\hline \multirow[t]{2}{*}{ Use napkin } & No & 8.5 \\
\hline & Yes & 8 \\
\hline \multirow[t]{2}{*}{ Pregnancy after 35} & Yes & 8 \\
\hline & No & 7.5 \\
\hline \multirow[t]{2}{*}{ Pap test } & No & 7.5 \\
\hline & Yes & 7 \\
\hline \multirow[t]{2}{*}{ Children after 35} & Yes & 7 \\
\hline & No & 6.5 \\
\hline \multirow[t]{2}{*}{ Takes hormone after menopause } & No & 6 \\
\hline & Yes & 6.5 \\
\hline \multirow[t]{3}{*}{ Take adequate fruit } & No & 6 \\
\hline & Yes & 5.5 \\
\hline & Above 60 & 5.5 \\
\hline \multirow[t]{3}{*}{ Age } & $46-60$ & 5.5 \\
\hline & $30-45$ & 5.25 \\
\hline & Below 30 & 5 \\
\hline \multirow[t]{3}{*}{ Oral contraception } & Yes & 5 \\
\hline & No & 4.5 \\
\hline & Undergraduate & 4 \\
\hline \multirow[t]{4}{*}{ Education } & Primary & 4.25 \\
\hline & Secondary & 4.25 \\
\hline & Illiterate & 4.5 \\
\hline & Above 60 & 4 \\
\hline \multirow[t]{3}{*}{ Age of husband } & $40-60$ & 3.65 \\
\hline & Below 30 & 3.8 \\
\hline & $30-45$ & 3.5 \\
\hline
\end{tabular}

used for anticipation. The compared notable features with weightage values have been displayed in Table 6. Finally, an algorithm has been developed based on the weightage values of Table 6 .

After analyzing the significances of the factor of cervical, ovarian, and stress we have derived an algorithm for predicting the risk levels of the diseases which is shown below, 


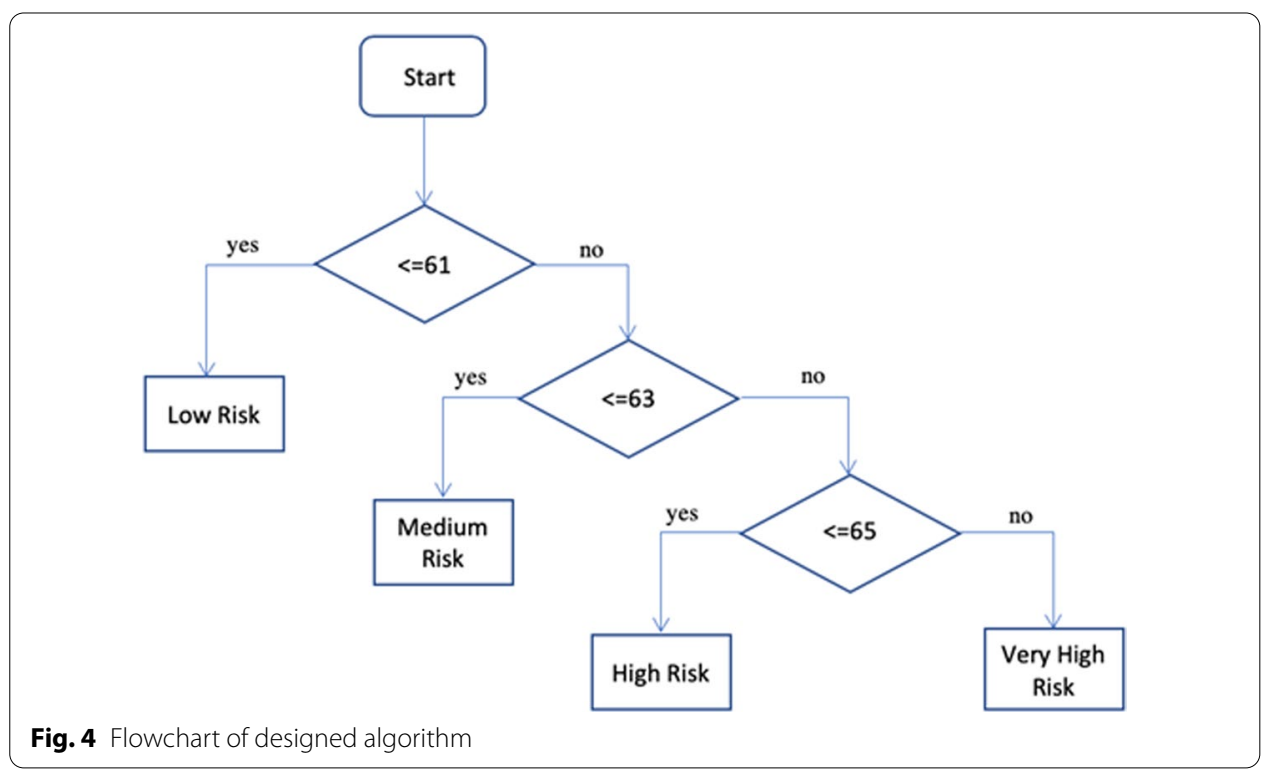

Step 1. Start

Step 2. read weights

Step 3. total_weights $\leftarrow \sum$ weights

Step 4. prediction_difference $\leftarrow$

Step 5. if total_weights $<=$ prediction_difference $+\sum$ lowest then print LOW RISK

Step 6. else if total_weights $<=$ (prediction_difference* 2 ) $+\sum$ lowest then print MEDIUM RISK

Step 7. else if total_weights $<=$ (prediction_difference ${ }^{*} 3$ ) $+\sum$ lowest then print HIGH RISK

Step 8. else print VERY HIGH RISK

Step 9. Stop

The flowchart of the algorithm has been shown in Fig. 4. With the help of the above algorithm, we found out the respective flowcharts for the diseases. At last, we put all the flowcharts and significant factors together to elicit the superior significant factors. Afterward combining cervical, ovarian, and stress factors we have drawn the flowchart for all of them. From those flowcharts and using the algorithm that predicts from the significant factors we have prepared an application shown in Figs. 5 and 6. The application is well prepared with the intention to store the future data in the cloud provided by the users and use that information for further investigation. We got better results with the sample data after training and testing of the models which makes us confident to use the for predicting those diseases. After utilizing the upcoming data, we will be able predict the diseases much more accurately.

The combined decision trees of cervical and ovarian cancer were pointed in Fig. 4, which indicates there are maximum chances to be infected by cervical virus if a woman had more than 2 children. If she had 1-2 children and her first intercourse was made when she was less than 16 years old than there are also maximum possibility of cervical 


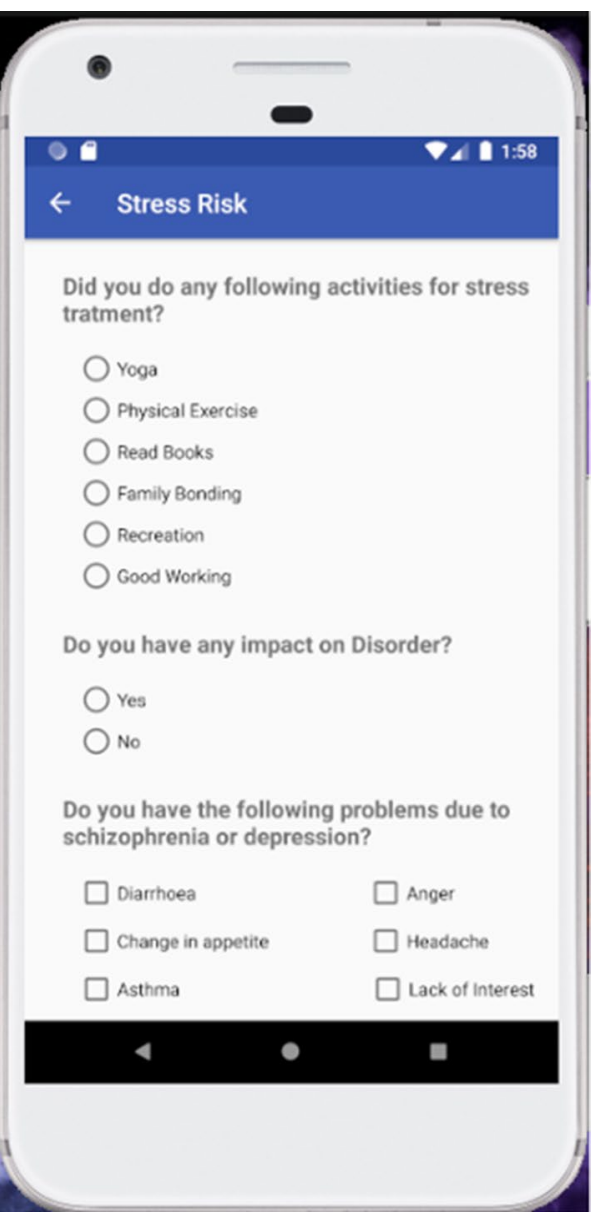

Fig. 5 Android application for cancer risk prediction (women health)

cancer. In here, a decision is made taking 6 precious factors for emerging cervical cancer. Likewise, taking 15 parameters a decision is made to find out the risk of appearing ovarian cancer. The most risk factors are abortion, age of husband, alcohol consumption, etc.

\section{Conclusion}

Cervical and ovarian cancers are the dominant causes of women's demise in Bangladesh. The majority of the people are unconscious of it. Death is inescapable because of cervical and ovarian cancer. From the findings, we got the evidence that immune response may be damaged due to neurodegenerative disease may even enhance the development of cancer. In this scrutiny, risk factors of cervical and ovarian cancer were analyzed carefully. Here, data mining and machine learning models like SVM, Random Forest, Logistics Regression, AdaBoost, Naïve Bayes, Neural Network, kNN, CN2 rule, Decision Tree, Quadratic Classifier have been used and those models were compared with two 


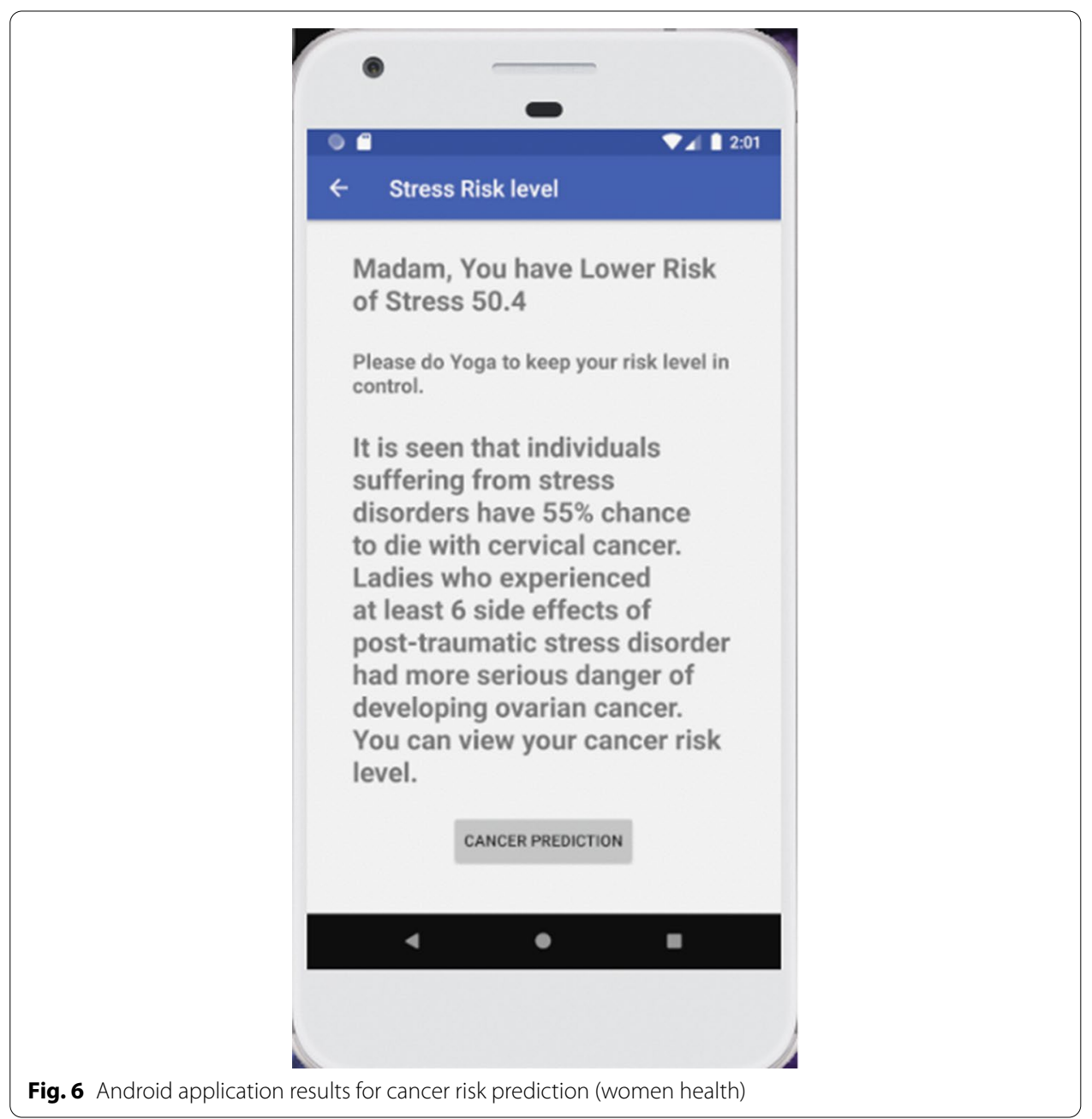

different tools. The obtained result for neurodegenerative disease shows that AdaBoost performed the best with a classification accuracy of $78.8 \%$ in orange and $79 \%$ in Sklearn. In the case of cervical cancer, Logistics Regression provides the best score of $84.8 \%$ and with Sklearn we've got $79.3 \%$. On the other hand, SVM shows the best accuracy of $88.3 \%$ in orange, and the decision tree provides $98.6 \%$ classification accuracy in Sklearn for ovarian cancer. Based on all the analyses finally, an algorithm along with a smart app was developed by the weightage values generated from the analysis. Future works can be done by improving the dataset size, tuning parameters, and more effective analysis.

\section{Summary of the work}

Cervical and ovarian cancer is the one of the most frightening disease among females in the low approaching nation like Bangladesh. The community of Bangladesh are lacking behind in education and awareness about these two cancers. Previous studies have found that stress is somehow influencing these two cancers. Any kind of prediction of cervical and ovarian cancer among Bangladeshi female's is not available in this modern age. Purpose: To find out the association between factors and the most significant factors of stress, cervical, ovarian cancer. Contribute a prediction on befalling cervical and 
ovarian cancer based on their worthy factors as well as stress parameters. Methods: A case control study has been made on 298 patients of cervical and 522 patients of ovarian cancer. Cases of 197 and control of 100 were considered for cervical cancer. In case of ovarian cases of 267 and control of 254 beheld for data mining analysis. For analyzing performance of several machine learning models e.g. Logistics Regression, Random Forest, AdaBoost, Naïve Bayes, Neural Network, kNN, CN2 rule Inducer, Decision Tree, Quadratic Classifier were compared with their standard metrics. For certainty info gain, gain ratio, gini index were revealed of both cervical and ovarian cancer. Attributes were ranked using different feature selection evaluators. Then the most significant analysis was made with the significant factors. Factors like children, age of first intercourse, age of husband, pap test, age are significantly higher factors of cervical cancer. On the other hand, genital area infection, pregnancy problem, use of drugs, abortion, number of children important factors of ovarian cancer. The analysis was made with significant factors of stress, cervical and ovarian cancer that will help us to predict the risk of occurring cervical or ovarian cancer and may help to abate the cancer not only from Bangladesh but all over the world. After analyzing a weightage table has been created to make an algorithm which can predict risk level of two fatal cancer of Women (cervical and Overian) along with mental health.

\section{Methods used in manuscripts}

All methods were carried out in accordance with relevant guidelines and regulations.

\section{Informed consent/waiver on informed consent}

The need for written informed consent was waived by the Institutional Review Board/ ethics committee of Department of Software Engineering, Daffodil International University. Because the Concern Hospitals dataset consisted of de-identified secondary data for research purposes. Verbal Consent from all the participants has been taken that no information will be disclosed and the dataset will only be used for research purpose.

\section{Supplementary Information}

The online version contains supplementary material available at https://doi.org/10.1186/s12859-021-04131-6.

Additional file 1. Sample Questioner for Cervical Cancer Data Collection.

Additional file 2. Sample Questioner for Ovarian Cancer Data Collection.

\section{Acknowledgements}

The authors are grateful to Dr. Nahid Farhana Chowdhury (Gynecologist) and Dr. Tonusree Chakraborty (Assistant Surgeon) to finalize the questionnaire and collect the dataset. The authors are grateful to those who have participated in this research and also grateful to those who are in case and control groups and provided their valuable information for conducting this research.

\section{Authors' contributions}

Sayed Asaduzzaman (Corresponding Author and First Author): Conceptualization; Methodology; Validation; Data Curation; Supervision; Md. Raihan Ahmed: Software; Methodology; Formal analysis; Writing —Original Draft; Hasin Rehana: Investigation; Writing_Original Draft; Formal analysis; Setu Chakraborty: Data Curation; Resources; Md. Shariful Islam: Data Curation; Resources; Touhid Bhuiyan: Writing — Review \& Editing; Project administration. All authors read and approved the final manuscript.

Funding

There was no funding for the research. 


\section{Declarations}

Ethics approval and consent to participate

This study was approved by the Institutional Review Board/ethics committee of Department of Software Engineering, Daffodil International University.

\section{Consent for publication}

Not applicable.

\section{Competing interests}

There is no conflict of interest among all the authors.

\section{Author details}

'Department of Computer Science and Engineering, Rangamati Science and Technology University, Vedvedi, Rangamati, Bangladesh. ${ }^{2}$ Department of Software Engineering, Daffodil International University, Dhanmondi, Dhaka, Bangladesh. ${ }^{3}$ Department of Computer Science and Engineering, Daffodil International University, Dhanmondi, Dhaka, Bangladesh. ${ }^{4}$ Department of Information and Communication Technology, Mawlana Bhashani Science and Technology University, Tangail 1902, Bangladesh. ${ }^{5}$ Department of Computer Science and Engineering, Rajshahi University Engineering and Technology, Rajshahi, Bangladesh. ${ }^{6}$ Department of Biotechnology and Genetic Engineering, Mawlana Bhashani Science and Technology University, Tangail, Bangladesh.

Received: 8 December 2020 Accepted: 7 April 2021

Published online: 24 April 2021

\section{References}

1. Seo J, Park M. Molecular crosstalk between cancer and neurodegenerative diseases. Cell Mol Life Sci. 2020;77(14):2659-80.

2. Cervical cancer. n.d. https://www.who.int/health-topics/cervical-cancer\#tab=tab_1. Accessed 16 Feb 2021.

3. PTSD Associated with Higher Risk of Ovarian Cancer | Cancer Network. n.d. https://www.cancernetwork.com/view/ ptsd-associated-higher-risk-ovarian-cancer. Accessed 16 Feb 2021.

4. Cancer. n.d. https://www.who.int/news-room/fact-sheets/detail/cancer. Accessed 16 Feb 2021.

5. Endometrial, Ovarian, and Cervical Cancer. n.d. https://www.clevelandclinicmeded.com/medicalpubs/diseaseman agement/womens-health/gynecologic-malignancies/. Accessed 16 Feb 2021

6. World Health Organization. 2008. Cervical cancer, human papillomavirus (HPV) and HPV vaccines: Key points for policy-makers and health professionals. World Health Organization.

7. Kuroda K, Yamamoto Y, Yanagisawa M, Kawata A, Akiba N, Suzuki K, Naritaka K. Risk factors and a prediction model for lower limb lymphedema following lymphadenectomy in gynecologic cancer: a hospital-based retrospective cohort study. BMC Womens Health. 2017;17(1):1.

8. Andersen BL. Predicting sexual and psychologic morbidity and improving the quality of life for women with gynecologic cancer. Cancer. 1993;71(S4):1678-90.

9. Kletetschka G, Bazala R, Takáč M, Svecova E. Magnetic domains oscillation in the brain with neurodegenerative disease. Sci Rep. 2021;11(1):1.

10. Kanehisa M, Furumichi M, Sato Y, Ishiguro-Watanabe M, Tanabe M. KEGG: integrating viruses and cellular organisms. Nucleic Acids Res. 2021;49(D1):D545-51.

11. Ueda K, Yanaihara N, lida Y, Kobayashi R, Kato S, Matsuda Y, Tomita K, Yamamura N, Saito R, Hirose S, Kuroda T. Analysis of risk factors for patients with early-stage cervical cancer: a study of 374 patients. J Obstetr Gynaecol Res. 2021:47(1):270-8

12. Okugawa K, Yahata H, Sonoda K, Kodama K, Yagi H, Ohgami T, Yasunaga M, Onoyama I, Kaneki E, Asanoma K, Kobayashi H. Evaluation of adjuvant chemotherapy after abdominal trachelectomy for cervical cancer: a singleinstitution experience. Int J Clin Oncol. 2021;26(1):216-24.

13. Steben M, Duarte-Franco E. Human papillomavirus infection: epidemiology and pathophysiology. Gynecol Oncol. 2007;107(2):S2-5.

14. Insinga RP, Dasbach EJ, Elbasha EH. Epidemiologic natural history and clinical management of Human Papillomavirus (HPV) disease: a critical and systematic review of the literature in the development of an HPV dynamic transmission model. BMC Infect Dis. 2009;9(1):1-26.

15. Soo RA, Stone EC, Cummings KM, Jett JR, Field JK, Groen HJ, Mulshine JL, Yatabe Y, Bubendorf L, Dacic S, Rami-Porta R. Scientific advances in thoracic oncology 2016. J Thorac Oncol. 2017;12(8):1183-209.

16. Roberts AL, Huang T, Koenen KC, Kim Y, Kubzansky LD, Tworoger SS. Posttraumatic stress disorder is associated with increased risk of ovarian cancer: a prospective and retrospective longitudinal cohort study. Can Res. 2019;79(19):5113-20.

17. Aslan D, Napuli J, Walters S. Association of chiropractic colleges research agenda conference 2020 abstracts of proceedings. J Chiropr Educ. 2020;34(1):66.

18. Teasley HE, Beesley A, Kim TH, Risinger J, Young SL, Jeong JW, Schammel DP, Lessey BA, Elder JW, Puls L. Differential expression of KRAS and SIRT1 in ovarian cancers with and without endometriosis. Reprod Sci. 2020;27(1):145-51.

19. Jayson GC, Kohn EC, Kitchener HC, Ledermann JA. Ovarian cancer. The Lancet. 2014;384(9951):1376-88.

20. Gaba F, Blyuss O, Liu X, Goyal S, Lahoti N, Chandrasekaran D, Kurzer M, Kalsi J, Sanderson S, Lanceley A, Ahmed M. Population study of ovarian cancer risk prediction for targeted screening and prevention. Cancers. 2020;12(5):1241. 
21. Sp N, Kang DY, Kim DH, Lee HG, Park YM, Kim IH, Lee HK, Cho BW, Jang KJ, Yang YM. Methylsulfonylmethane inhibits cortisol-induced stress through p53-mediated SDHA/HPRT1 expression in racehorse skeletal muscle cells: a primary step against exercise stress. Exp Ther Med. 2020;19(1):214-22.

22. Colon-Echevarria CB, Ortiz T, Maldonado L, Hidalgo-Vargas MJ, Pérez-Morales J, Aquino-Acevedo AN, HerreraNoriega R, Bonilla-Claudio M, Castro EM, Armaiz-Pena GN. Zoledronic acid abrogates restraint stress-induced macrophage infiltration, PDGF-AA expression, and ovarian cancer growth. Cancers. 2020;12(9):2671.

23. Twombly R. Cancer surpasses heart disease as leading cause of death for all but the very elderly. J Natl Cancer Inst. 2005;97(5):330-1.

24. Cassidy A, Duffy SW, Myles JP, Liloglou T, Field JK. Lung cancer risk prediction: a tool for early detection. Int J Cancer. 2007;120(1):1-6

25. Rebbeck TR, Burns-White K, Chan AT, Emmons K, Freedman M, Hunter DJ, Kraft P, Laden F, Mucci L, Parmigiani G, Schrag D. Precision prevention and early detection of cancer: fundamental principles. Cancer Discov. 2018;8(7):803-11.

26. Gordon R. Skin cancer: an overview of epidemiology and risk factors. In: Seminars in oncology nursing 2013 Aug 1 (Vol. 29, No. 3, pp. 160-169). WB Saunders.

27. Azad K. Type 1 diabetes: the Bangladesh perspective. Indian J Endocrinol Metabol. 2015;19(Suppl 1):S9.

28. Patel J, TejalUpadhyay $D$, Patel $S$. Heart disease prediction using machine learning and data mining technique. Heart Dis. 2015;7(1):129-37.

29. Scikit-learn: Machine learning in Python—Scikit-learn 0.24.1 documentation. (n.d.). https://scikit-learn.org/stable/. Accessed 16 Feb 2021

30. Kumar UK, Nikhil MS, Sumangali K. Prediction of breast cancer using voting classifier technique. In: 2017 IEEE international conference on smart technologies and management for computing, communication, controls, energy and materials (ICSTM); IEEE. 2017. p. 108-14.

31. Shouman M, Turner T, Stocker R. Using decision tree for diagnosing heart disease patients. In: Proceedings of the ninth Australasian data mining conference-volume 121 2011. p. 23-30.

32. Parthiban R, Usharani S, Saravanan D, Jayakumar D, Palani DU, StalinDavid DD, Raghuraman D. Prognosis of chronic kidney disease (CKD) using hybrid filter wrapper embedded feature selection method. Eur J Mol Clin Med. 2021;7(9):2511-30.

\section{Publisher's Note}

Springer Nature remains neutral with regard to jurisdictional claims in published maps and institutional affiliations.

- fast, convenient online submission

- thorough peer review by experienced researchers in your field

- rapid publication on acceptance

- support for research data, including large and complex data types

- gold Open Access which fosters wider collaboration and increased citations

- maximum visibility for your research: over $100 \mathrm{M}$ website views per year

At BMC, research is always in progress.

Learn more biomedcentral.com/submissions 